[Agr. Biol. Chem., Vol. 36, No. 11, p. 1955 1960, 1972]

\title{
Photochemical Reaction between Uridine and Sulfhydryl Compounds
}

\author{
By Hideo Ochial and Hitoshi Shibata \\ Laboratory of Biochemistry, College of Agriculture, \\ Shimane University, Matsue, Shimane, Japan
}

Received April 5, 1972

\begin{abstract}
Kinetics of photochemical reaction between uridine and sulfhydryl compounds were investigated in phosphate buffer $(\mathrm{pH} 7.0)$ and in unbuffered aqueous solution under aerobic condition. The results obtained clearly demonstrate that the photo-induced hydration or reduction of the uridine molecule was significantly influenced by the amount of sulfhydryl group present in the reaction medium. Reaction on uridine $(1 \mathrm{~mm})$ was observed to lead to photohydration with pseudo first order rates, independently of the presence of cysteine ( $1 \mathrm{~mm}$ or $2 \mathrm{~mm}$ ), while in the presence of dithiothreitol (DTT, $1 \mathrm{~mm}$ to $10 \mathrm{~mm}$ ) both photoreduction and photohydration of uridine were observed. The rate of photoreduction came to predominate as the amount of DTT increased. The reaction was discussed from the view point of food chemistry as well as reaction pathways.
\end{abstract}

From the view point of food chemistry it was demonstrated that uracil was decomposed by the ultraviolet ray irradiation in the presence of a small amount of hydrogen peroxide to give parabanic acid with concurrent formation of less stable oxaluric acid, which was readily hydrolyzed to oxalic acid and urea." However, it is also important to investigate the photochemical behavior of nucleic acid constituents under "heterogenous" or mixed condition, because foods are originally heterogenous ones. This prompted an investigation of the photochemical kinetics of mixed solution of uridine together with sulfhydryl compounds containing cysteine or dithiothreitol (DTT). The present report describes that reactions are significantly influenced by the property and amount of sulfhydryl group present in the reaction medium.

\section{MATERIALS AND METHODS}

Uridine was obtained from Kojin Co. Ltd., Tokyo. Cysteine and dithiothreitol were purchased from Nakarai Co., Ltd., Kyoto. The reagents were used

Radical Reaction of Food Constituents (2). without further purification. Reaction solution contained uridine $(1 \mathrm{~mm})$ and various concentration of sulfhydryl compounds in $0.1 \mathrm{M}$ phosphate buffer ( $\mathrm{pH}$ 7.0), and in unbuffered aqueous solution. The solution is kept in an open Petri dish at $0.25 \mathrm{~cm}$ depth and irradiated $12 \mathrm{~cm}$ apart from low pressure mercury resonance lamps (Mitsubishi GKL 101, 10W 3) at room temperature under aerobic conditions. Disappearence of uridine was followed by the measurement of absorption maximum at $262 \mathrm{~nm}$. 5,6-Dihydrouridine was determined by orcinol assay $(660 \mathrm{~nm})$ using D-ribose as a standard specimen. Color development in the orcinol assay was influenced by the presence of DTT. However, this could be compensated by appropriate correction factors which were gained from the assay procedure in the presence of DTT of each concentration. Formation of 5;6-dihydro 6-hydroxy uridine (uridine hydrate) was followed by taking advantage of the reversibility of the hydrate into uridine by heat-treatment $\left(90^{\circ} \mathrm{C}, 20 \mathrm{~min}\right)$ in acidic condition ( $\mathrm{pH}$ 1). Both cysteine and cystine were estimated simultaneously by polarographic method using $0.0 \mathrm{~V}$ as half-wave potential for cysteine and $-0.45 \mathrm{~V}$ for cystine. Interestingly maximum wave at the cystine determination was perfectly eliminated in the presence of uridine used in the experiment and also in the presence of uridine hydrate produced in the course of the reaction. Reduced form of DTT was determined colorimetrically after the Ellman's method 2 using 5,5'-dithiobis(2-nitrobenzoic acid) as a color re- 
agent (412 nm) and oxidized form of DTT (intramolecular disulfide of DTT) was estimated optically at the absorption maximum $283 \mathrm{~nm}(\varepsilon=273, \mathrm{pH} \mathrm{7.0})$. Authentic 5,6-dihydrouridine was synthesized after the method of Cerutti et al. ${ }^{\text {s) }}$ Identification of photochemically produced substances was made with direct comparisons with authentic specimens on paper chromatography and on thin-layer chromatography using the following solvents: (1) n-butanol-water $(86: 14)$, (2) n-butanolacetic acid-water $(4: 1: 5),(3) n$-butanol-ethanol-water $(9: 9: 1)$, (4) $n$-butanol-25\% $\mathrm{NH}_{4} \mathrm{OH}(10: 1)$.

\section{RESULTS}

The photochemical reaction of the mixed unbuffered aqueous solution of uridine $(1 \mathrm{~mm})$ and cysteine $(1 \mathrm{~mm})$, followed at $262 \mathrm{~nm}$, was light-dose dependent, pseudo first order with $k=3.31 \quad 10^{-2} / \mathrm{min}$. Irradiation of the solution to the point where $90 \%$ of the uridine was disappeared led to the production of uridine hydrate as a sole photoproduct. The reaction product was analyzed and identified on the thin-layer chromatography (silica gel $\mathrm{GF}_{254}$ ) using the solvents as described in "Materials and Methods." Neither 5,6-dihydrouridine nor uridine-cysteine complex was detectable in the photoproduct. On the other hand, $68 \%$ of cysteine remained unchanged and $14 \%$ was converted to cystine independently of the presence of uridine. The experiment using $2 \mathrm{~mm}$ cysteine bring about the similar results. Therefore it is concluded that photochemical hydration of uridine proceeded predominantly in the experimental condition employed. This appears to be contrary to Smith's results which demonstrated the formation of uracil-cysteine complex by irradiating a "concentrated" solution of uracil together with cysteine hydrochloride." This discrepancy is supposed to result from the differences of both quantum yields and nature of reaction for uracil and uridine irradiated in aqueous solution, respectively; quantum yield for uracil was estimated to be 5.2 and for uridine $21.6^{51}$
Table I. Identification of the Product by THIN-LAyer Chromatography ON SiltCa Gel GF $_{254}$

Color reagent for 5,6-dihydrouridine: the Anisaldehyde reagent ${ }^{12}$, and the Fink's reagent. ${ }^{13}$ Uridine was detected as the dark spot shown on UV. illumination in dark room and also by the anisaldehyde reagent. Uridine hydrate was detected through the recovery into the UV-absorbing uridine by $10 \%$ acetic acid (spraying) followed by heattreatment and also by the anisaldehyde reagent. Uridine-cysteine complex was positive for both the anisaldehyde and ninhydrin reagent. The product which was a sample isolated from the photochemical reaction mixture between uridine and DTT was positive for both the anisaldehyde and the Fink's reagent.

\begin{tabular}{|c|c|c|c|c|c|}
\hline Solvent & $\begin{array}{l}\text { Pro- } \\
\text { ducta: }\end{array}$ & $\begin{array}{c}5,6- \\
\text { Dihydro } \\
\text { uridine }\end{array}$ & Uridine & $\begin{array}{l}\text { Uridine } \\
\text { hydrate }\end{array}$ & $\begin{array}{l}\text { Uridine- } \\
\text { Cysteine } \\
\text { complex }\end{array}$ \\
\hline (1) & 0.26 & 0.26 & 0.48 & 0.23 & \\
\hline (2) & 0.40 & 0.40 & 0.54 & & 0.10 \\
\hline (3) & 0.56 & 0.56 & 0.71 & 0.52 & 0.15 \\
\hline (4) & 0.11 & 0.11 & 0.18 & & 0.05 \\
\hline
\end{tabular}

In one experiment using dimercapto compound DTT $(1 \mathrm{~mm})$ in the place of cysteine, however, it was found that 5,6-dihydrouridine was formed as one of the photoproducts. For the purpose of isolation of 5,6-dihydrouridine formed, the mixed solution of uridine $(1 \mathrm{~mm})$ and DTT $(10 \mathrm{~mm})$ was irradiated to the point where $10 \%$ of starting uridine remained unchanged. The reaction mixture (1 liter) was concentrated under reduced pressure in a rotary evaporator, filtered off and lyophilized. Another photoproduct, uridine hydrate, was almost completely converted into uridine during the course of this treatment. The amorphous powder obtained was chromatographed over silica gel column using n-butanol-water $(86: 14)$ as developing solvent. Aliquots which were positive in orcinol assay were collected in one portion and the combined solution was lyophilyzed to give white powder. The purification by silica gel column chromatography was repeated until the product was completely free from uridine and 
DTT. The powder obtained $(80 \mathrm{mg}, 33 \%$ in yield) was identified as 5,6-dihydrouridine from the behaviors on concurrent development with authentic specimen on silica gel thin-layer using four kinds of developing solvents. Table I shows the $R f$ values of the product, authentic 5,6-dihydrouridine and related compounds. Both IR and NMR spectra of the product were found to coincide with those of the authentic 5,6-dihydrouridine.

Table II. Half-life Periods of IRRADIATED URIDINE

\begin{tabular}{|c|c|c|c|}
\hline $\begin{array}{l}\text { Medium } \\
\text { DTT }\end{array}$ & $\begin{array}{c}\text { Phosphate } \\
\text { Buffer } \\
\text { (pH 7.0, } \\
0.1 \mathrm{M}) \\
\end{array}$ & Unbuffered & $\begin{array}{l}\text { Acidic } \\
(\mathrm{pH} 1)\end{array}$ \\
\hline $0 \mathrm{mM}$ & $20 \min$ & $19 \mathrm{~min}$ & $14 \mathrm{~min}$ \\
\hline $1 \mathrm{~mm}$ & $15^{a !}$ & $13^{a}$ & $9^{a 1}$ \\
\hline $2 \mathrm{~mm}$ & $12^{a)}$ & $11^{\alpha}$ & $8^{a t}$ \\
\hline $5 \mathrm{~mm}$ & $10^{a 1}$ & $10^{a i}$ & $5^{a\}}$ \\
\hline $10 \mathrm{~mm}$ & $8^{01}$ & $9^{a}$ & $6^{a 1}$ \\
\hline
\end{tabular}

a) Apparent half-life period. See the text for the details.

Table III. Production of Uridine-hydrate and Orcinol Reaction Positive Substance(s) AFter 30 MIN-IRRADIATION IN THE Phosphate Buffer (pH 7.0, 0.1 M)

\begin{tabular}{ccc}
\hline DTT & Uridine-hydrate & $\begin{array}{c}\text { Orcinol reaction } \\
\text { positive substance(s) }\end{array}$ \\
\hline $0 \mathrm{mM}$ & 54 mole $\%$ & 25 mole $\%$ \\
1 & 48 & 45 \\
2 & 44 & 60 \\
5 & 40 & 66 \\
10 & 33 & 65 \\
\hline
\end{tabular}

Uridine $(1 \mathrm{~mm})$ in $0.1 \mathrm{M}$ phosphate buffer ( $\mathrm{pH} 7.0)$ is converted by UV-irradiation with half life of $20 \mathrm{~min}$ as light-dose dependent pseudo first order kinetics under aerobic condition in the absence of sulfhydryl compound. In the presence of DTT, the photochemical conversion of uridine run no longer as pseudo first order one. However, as a matter of convenience, the apparent half life periods (the time needed for the disappearance of half amount of starting uridine) of irradiated uridine in the presence of various amount of DTT were shown in Table II, which demonstrated that the photochemical conversion of uridine was significantly accelarated by the presence of DTT. The similar results were observed in the unbuffered and in acidic aqueous solution ( $\mathrm{pH} 1$, sulfuric acid). Table III shows the production of uridine hydrate and orcinol reaction positive substances (predominantly 5,6-dihydrouridine), in the presence of various amount of DTT, after $30 \mathrm{~min}$ irradiation in $0.1 \mathrm{M}$ phosphate buffer. It is obvious that the more presence of DTT exerted the more production of 5,6-dihydrouridine and the lesser of uridine hydrate.

Figure 1 shows the time course of the photochemical conversion of uridine in the

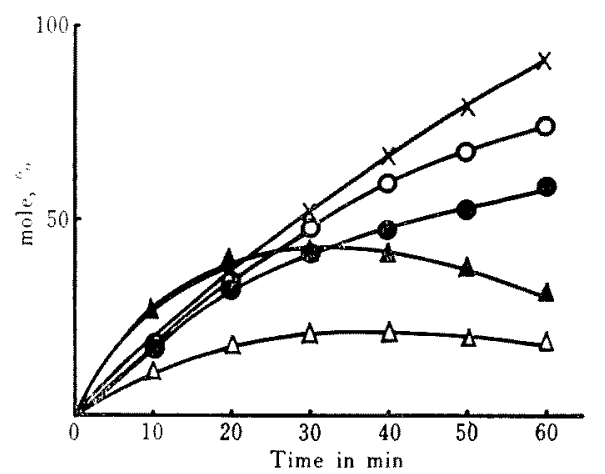

Fig. 1. Time Course of Photochemical Conversion of Uridine.

Production of uridine hydrate $x-x$ in the absence of DTT.

Production of uridine hydrate $0-0$

Production of orcinol reaction $\triangle-\triangle$ positive substance(s) in the presence of DTT (1 mm).

Production of uridine hydrate

Production of orcinol reaction positive substance(s)

in the presence of DTT ( $5 \mathrm{~mm})$.

absence and in the presence of DTT, where the net production of 5,6-dihydrouridine was demonstrated from that the found value with 
DTT minus the value without DTT at each assay. It has been shown that the positively found value in the absence of DTT was resulted from the hydrolysis of the nucleoside bond of uridine hydrate before the conversion of the hydrate into uridine which was stable on acid treatment. This photoreduction was found to follow second order rate kinetics, for instance, with a rate constant $k=4.2 \times$ $10^{-3} \mathrm{l} / \mathrm{mole} \cdot \mathrm{sec}$. in the presence of $10 \mathrm{~mm}$ DTT, especially at the early stage. It is observed, however, that the accumulation of 5,6-dihydrouridine was diminished as time proceeded, perhaps due to decomposition of the formed dihydrouridine and not due to decreased amount of DTT.

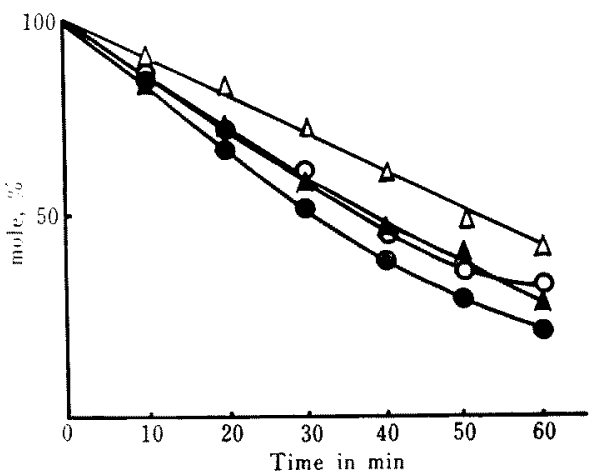

Fig. 2. Time Course of Photochemical Conversion of DTT in the Absence and in the Presence of Uridine (I mm).

O-O DTT (1 mM) without uridine; - DTT (1 $\mathrm{mm})$ with uridine; $\triangle-\triangle$ DTT $(2 \mathrm{~mm})$ without uridine; $\Delta$ DTT $(2 \mathrm{~mm})$ with uridine.

DTT in the phosphate buffer solution decreased just like pseudo first order with $k=$ $1.82 \times 10^{-2} / \mathrm{min}$ with $1 \mathrm{~mm}$ DTT present, which was smaller than that of uridine disappearance. The transformation of DTT was also accelerated by the presence of uridine, as shown in Fig. 2, to give oxidized DTT (intramolecular disulfide of DTT as a main product and perhaps some intermolecular disulfides).

Table IV shows the change of $\mathrm{pH}$ during
Table IV. Changes in pH during UV. IRRADIATION TO URIDINE-DTT UNBUFFERED Aqueous Solution

\begin{tabular}{|c|c|c|c|c|}
\hline $\begin{array}{l}\text { Time in min } \\
\text { DTT }\end{array}$ & 0 & 20 & 40 & 60 \\
\hline $0 \mathrm{~mm}$ & 5.0 & 5.2 & 5.2 & 5.1 \\
\hline 1 & 5.7 & 5.4 & 5.3 & 4.8 \\
\hline 2 & 5.8 & 5.4 & 5.1 & 4.8 \\
\hline 5 & 5.6 & 5.4 & 5.2 & 4.5 \\
\hline 10 & 5.9 & 6.0 & 5.6 & 5.3 \\
\hline
\end{tabular}

UV irradiation to uridine-DTT unbuffered aqueous solution. Changes in $\mathrm{pH}$ are very little in the absence of DTT, whereas $\mathrm{H}^{+}$ concentration increased as about 10 times as the initial value during the reaction process in the presence of $1 \mathrm{~mm}, 2 \mathrm{~mm}$ and $5 \mathrm{~mm}$ DTT, respectively. Such changes in $\mathrm{pH}$ would influence both the velocity of the photochemical reactions and the ratio of photoproducts (Table II). ${ }^{6}$ However, the changes in $\mathrm{pH}$ might be more noted from the standpoint of food chemistry.

\section{DISCUSSION}

The results of the experiments described in this paper clearly demonstrate that the photoinduced hydration or reduction of the uridine molecule was significantly influenced by the property and amount of sulfhydryl compound present in the reaction medium. In the presence of $2 \mathrm{~mm}$ monomercapto compound cysteine ( $2 \mathrm{~mm} \mathrm{SH-group)} \mathrm{the} \mathrm{reaction} \mathrm{was}$ observed to proceed predominantly to give uridine hydrate as a sole product. On the other hand, both photoreduction and photohydration of uridine were observed in the presence of $1 \mathrm{mM}$ dimercapto compound DTT (2 mM SH-group). Furthermore, the former reaction came to predominate as the amount of DTT increased. Neither uridine-dimer nor uridine-DTT complex was detected in the photoproducts obtained from the reaction 
between uridine $(1 \mathrm{~mm})$ and DTT (1 $\mathrm{mm}$ to $10 \mathrm{~mm}$ ). Conversion of DTT also was observed to be accelerated by the presence of uridine to give oxidized DTT as a main product. The photohydration of uridine might not be a serious problem from the view point of food chemistry because of the reversibility of the hydrate into starting material uridine by heat-treatment, whereas the photoreduction of uridine in the presence of sulfhydryl compounds should be noted as well as the fate of sulfhydryl compounds in the foods.

Originally the photochemical reaction between nucleic acid components and sulfhydryl compounds was studied from the standpoint of biochemistry. In 1962, Smith reported that the increased sensitivity of $E$. coli to killing by UV-light and the changes in sensitivity as a function of temperature during irradiation have been correlated with the increased production of DNA-protein crosslinking.7) Smith and his coworker could demonstrate that uracil and cysteine combined to form 5-s-cysteinyl 5,6-dihydro uracil by irradiating a "concentrated" solution of uracil-2- ${ }^{14} \mathrm{C}(2.3 \mathrm{~mm})$ and cysteine hydrochloride (10 mM) with UV light." 5,6Dihydro uracil was also found firstly as one of the photoproducts in that experiment.

Photoreduction of uridine in the presence of excess sodium borohydride was reported by Cerutti et al. to give 5,6-dihydrouridine. ${ }^{3,8}$ Jellinek and Johns reported a detailed study on the mechanisms of the photochemical addition of cysteine to uracil with concurrent formation of dihydrouracil. ${ }^{9}$ They proposed the mechanism of the reaction to proceed through the triplet excited state of uracil which could be quenched by oxygen molecule. Cerutti and Miller presented strong evidence of the uridine hydrate as to be 5,6-dihydro 6-hydroxy uridine, ${ }^{10}$ and Burr et al. described that photohydration of uracil included reaction between a singlet excited uracil molecule and a neutral water molecule. ${ }^{6}$.

Based upon these considerations and our results, possible reaction mechanisms of uridine together with DTT may be proposed as shown in Fig. 3. With UV-irradiation, excited singlet state of uridine is formed which is very short-lived and can combine only a water molecule, in particular, so easily in the aqueous solution employed in our experiments, and give rise to uridine hydrate (I) Some parts of the excited singlet state can be translated into a triplet state which is relative-

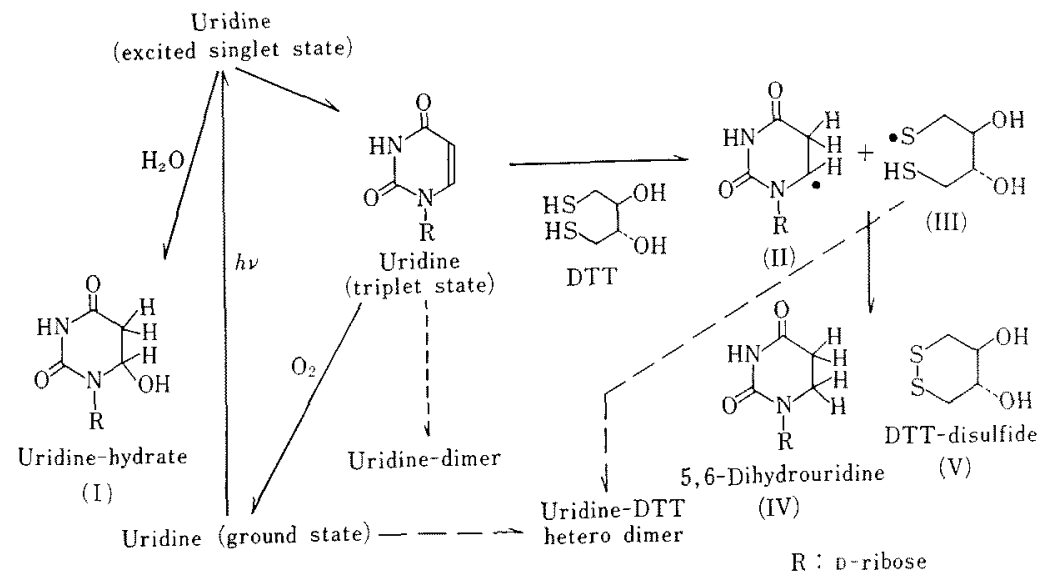

FIG. 3. Possible Reaction Mechanisms between Uridine and DTT. 
ly long-lived, perhaps a millisecond or longer. Through this triplet state, uridine homodimer could be produced if uridine was present enough dense. However, the dimer was not formed under the condition employed (uridine $1 \mathrm{~mm}$ ). Furthermore, it has been shown, in fact, that molecular oxygen effectively quenches the triplet excitation of uridine and thus inhibits the reaction through the triplet state. Therefore, in the absence or the presence of only a small amount of sulfhydryl compound, the photochemical reaction under aerobic condition results in the photohydration predominantly.

On the other hand, when DTT, an effective $\mathrm{SH}$-protector, is present in the reaction medium, DTT would eliminate the effect of oxygen to contribute to maintain the triplet state of uridine in the medium: in water saturated with air at room temperature, the concentration of oxygen is about $0.2 \mathrm{~mm}^{1{ }^{11}}$ Thus uridine triplet can abstract an $\mathrm{H}$ atom from one of sulfhydryl group of DTT to give both hydrogen addition radical (II) and thiyl DTT radical (III). The former may react with another sulfhydryl group of the same DTT radical to yield 5,6-dihydrouridine (IV), and the latter give rise to DTT-disulfide (V). Hence dimercapto compound, DTT, should be prefered stereochemically to monomercapto compound, cysteine, for the promotion of the photoreduction. From the similar point of view, it should be hardly expected that thiyl DTT radical could react with ground state of uridine to yield uridine-DTT hetero dimer.

Under nitrogen atmosphere, a considerable formation of 5,6-dihydrouridine was also observed by irradiating a mixed aqueous solution of uridine together with a dimercapto compound. Details will be described elsewhere.

Acknowledgement. The author wishes to express his sincere thanks to Prof. Y. Sato and Prof. M. Nami$k i$, Nagoya University, for their encouragement. Thanks are also due to Dr. Y. Matsui, Shimane University, for running the polarography and also due to Mr. H. Hosogai and Mr. H. Sumi for their technical assistances. This work was supported in part by a grant from the Ministry of Education.

\section{REFERENCES}

1) H. Ochiai, Agr. Biol. Chem, 35, 622 (1971).

2) G. L. Ellman, Arch. Biochem. Biophys, 82, 70 (1959).

3) P. A. Cerutti, Y. Kondo, W. R. Landis and B. Witkop, J. Am. Chem. Soc., 90, 771 (1968).

4) K. C. Smith and R. T. Aplin, Biochemistry, 5, 2125 (1966).

5) A. D. McLaren and D. Shugar, "Photochemistry of Proteins and Nucleic Acids," Pergamon Press, New York, N.Y. 1964, p. 180.

6) J. G. Burr, B. R. Gordon and E. H. Park, Photochem. Photobiol., 8, 73 (1968).

7) K. C. Smith, Biochem. Biophys. Res. Commun, 8, 157 (1962).

8) P. A. Cerutti, K. Ikeda and B. Witkop, J. Am. Chem. Soc., 87, 2505 (1965).

9) T. Jellinek and R. B. Johns, Photochem, Photobiol., 11, 349 (1970).

10) N. Miller and P. A. Cerutti, Proc. Natl. Acad. Sci. U.S., 59, 34 (1968).

11) R. K. Clayton, "Light and Living Matter," Vol. 2 , The Biological Part, McGraw-Hill, New York, N.Y., 1971, p. 210.

12) E. Stahl and U. Kaltenbach, $J$. Chromatogr., 5, 351 (1961).

13) R. M. Fink, R. E. Cline, Ch. McGaughey and K. Fink, Anal. Chem., 28, 4 (1956). 